\title{
Natural variation in maternal sensitivity Is reflected in maternal brain responses to infant stimuli
}

DOI:

10.1037/bne0000161

\section{Document Version}

Accepted author manuscript

Link to publication record in Manchester Research Explorer

\section{Citation for published version (APA):}

Elmadih, A., Wan, M. W., Downey, D., Elliott, R., Swain, J. E., \& Abel, K. M. (2016). Natural variation in maternal sensitivity Is reflected in maternal brain responses to infant stimuli. Behavioral neuroscience, 130(5), 500-510. https://doi.org/10.1037/bne0000161

\section{Published in:}

Behavioral neuroscience

\section{Citing this paper}

Please note that where the full-text provided on Manchester Research Explorer is the Author Accepted Manuscript or Proof version this may differ from the final Published version. If citing, it is advised that you check and use the publisher's definitive version.

\section{General rights}

Copyright and moral rights for the publications made accessible in the Research Explorer are retained by the authors and/or other copyright owners and it is a condition of accessing publications that users recognise and abide by the legal requirements associated with these rights.

\section{Takedown policy}

If you believe that this document breaches copyright please refer to the University of Manchester's Takedown Procedures [http://man.ac.uk/04Y6Bo] or contact uml.scholarlycommunications@manchester.ac.uk providing relevant details, so we can investigate your claim.

\section{OPEN ACCESS}


Natural Variation in Maternal Sensitivity is Reflected in Maternal Brain

\section{Responses to Infant Stimuli}

\section{Authors}

${ }^{\text {a, } 1}$ Alya Elmadih

${ }^{\text {a }}$ Ming Wai Wan

${ }^{\mathrm{b}}$ Darragh Downey

${ }^{\mathrm{b}}$ Rebecca Elliott

${ }^{\mathrm{c}}$ James E. Swain

${ }^{a}$ Kathryn M. Abel

\section{Affiliations, and author addresses}

${ }^{a}$ Centre for Women's Mental Health, Institute of Brain, Behaviour and Mental Health,University of Manchester, Manchester, M13 9PL, UK.

${ }^{b}$ Neuroscience and Psychiatry Unit, Institute of Brain, Behaviour and Mental Health, University of Manchester, Manchester, M13 9PL, UK.

${ }^{c}$ Department of Psychiatry, Psychology, Center for Human Growth and Development, Women and Infants Mental Health Program, University of Michigan, 4250 Plymouth Rd, Ann Arbor, MI 48109, USA.

\section{${ }^{1}$ Corresponding Author}

Alya Elmadih

$3^{\text {rd }}$ Floor East Jean McFarlane Building

University of Manchester

M13 9PL

UK

+441612750714/32

E-mail address: alyamadeh1@gmail.com

Number of words in the manuscript (including references: 7672)

Number of words in the abstract: 228

Number of figures: 2

Number of tables: 2 


\begin{abstract}
Background: Increasing evidence suggests that discrete neural networks which mediate emotion processing are activated when mothers respond to infant's images or cries.

Accumulating data also indicate that natural variation in maternal caregiving behavior is related to maternal oxytocin (OT) levels. However, brain activation to infant cues has not been studied comparing mothers at disparate ends of the 'maternal sensitivity' spectrum.
\end{abstract}

Methods: Based on observed mother-infant play interaction at 4-6 months postpartum in 80 antenatally-recruited mothers, 15 mothers with the highest sensitivity (HSMs) and 15 mothers with the lowest sensitivity (LSMs) were followed at 7-9 months using functional magnetic resonance imaging to examine brain responses to viewing videos of their 'own' versus an 'unknown' infant in three affect states (neutral, happy and sad). Plasma OT measurements were taken from mothers following play interactions with their infant. Results: Compared to LSMs, HSMs showed significantly greater brain activation in right superior temporal gyrus (STG) in response to own versus unknown neutral infant and to own happy versus neutral control. Changes in brain activation were significantly negatively correlated with plasma OT responses in HSMs mothers. Conversely, compared to HSMs, LSMs showed no significant activation difference in response to own infant separately or in contrast to unknown infant. Conclusion: Activation of STG may index sensitive maternal response to own infant stimuli. Sensitive parenting may have its unique profile in relation to brain responses which can act as biomarkers for future intervention studies that enhance sensitivity of maternal care.

Key words: fMRI: functional magnetic resonance imaging, BOLD: blood oxygenation level dependent response 


\section{Introduction}

It is now well established that maternal sensitivity - i.e. the degree to which a mother responds to her infant's signals in an appropriate and timely manner (Ainsworth et al., 1978) - plays an important role in supporting the infant's secure attachment formation, social functioning, emotional self-regulation and cognitive and language competences (Bigelow et al., 2010; Crosnoe et al., 2010; Pearson et al., 2011; Warren \&Simmens, 2005). Evidence that low maternal sensitivity heralded negative infant social, emotional and cognitive development (Alink et al., 2008; Downer \&Pianta, 2006; Kochanska\& Kim, 2012) has generated many interventions to enhance maternal sensitivity (Barlow et al., 2008). However, we are only beginning to understand mechanisms of change (Sandler et al., 2011) and, whether/how the neurobiology underlying maternal care changes following early interventions (BakermansKranenburg\& Van Ijzendoorn, 2015).

fMRI has demonstrated selective maternal BOLD activations (brain responses)to own infant faces or infant cries (Lorberbaum et al., 2002; Noriuchi et al., 2008; Ranote et al., 2004; Wan et al., 2014). This network of maternal brain responses includes areas involved in motivational and reward pathways (e.g. anterior cingulate and prefrontal cortices)(Seifritz et al., 2003); emotion processing (e.g. amygdala); decoding emotional memories (e.g. hippocampus) (Phelps et al., 2004); and areas implicated in secretion of the mammalian neuropeptide, oxytocin (OT) (a key modulator of social behavior- Lee et al., 2009) e.g. hypothalamus (Lenzi et al., 2009; Ranote et al., 2004; Swain et al., 2008).

Recently, maternal brain responses to infant stimuli, have been examined in relation to observed quality of maternal caregiving (Atzil et al. 2011, Musser et al., 2012). Our recent study in 20 healthy mothers (Wan et al., 2014) reported that positive mother-infant interaction (less directive maternal behavior and more attentive infant behavior (as opposed to directive 
and less attentive respectively)) was significantly associated with greater activation on viewing own vs. unknown infant especially in middle frontal gyrus. In 'synchronous' $(\mathrm{N}=13)$ compared to 'intrusive' mothers $(\mathrm{N}=10)$, Atzil et al. (2011) showed more BOLD activation in regions for response/salience and regulation (superior temporal gyrus (STG) and inferior frontal gyrus (IFG)), suggesting the importance of balanced responses for sensitive caregiving. Musser et al. (2012) rated mothers (N=22) for 'sensitivity', 'harmony' and 'intrusive' behavior with their infant. More sensitive behavior was associated with greater activation in areas implicated in response inhibition (frontal pole); areas for 'reading others' minds' (inferior frontal gyrus). Behavior with more harmony activated areas involved in recalling of memories (left hippocampal regions). By contrast, more intrusive maternal behavior activated areas implicated in possessing empathy with loved ones (left insula). Sensitive parenting in breastfeeding $(\mathrm{N}=9)$ and formula-feeding $(\mathrm{N}=8)$ mothers correlated with brain activity in amygdala and frontal cortex(Kim et al., 2011). The range of BOLD activation patterns reported by these studies in response to infant stimuli suggests that maternal behavior is a composite of multiple behaviors, with discrete maternal brain activation in relation to each aspect of behavior (Musser et al., 2012).

Maternal brain responses have also been studied in relation to maternal plasma OT levels, previously implicated in promoting high quality parenting (Feldman et al., 2007, 2010). Plasma OT levels have been reported as positively correlated with BOLD activation in brain areas linked to motivation (e.g. nucleus accumbens) in 'synchronous', but not in 'intrusive' mothers (Atzil et al., 2011). In spite of promising evidence linking OT to maternal behavior (Gordon et al., 2010; Feldman et al., 2007) and to brain responses (Atzil et al., 2011;

Strathearn et al., 2009), only one study to date has considered grouping mothers according to their parenting style, i.e. 'intrusiveness' or 'synchronous' behavior (Atzil et al., 2011) before 
examining the association between OT and brain responses. In our view, sensitive responsiveness should be considered as a 'comprehensive' concept based on the reciprocal relationship between mother and infant, rather than as fine grained behaviors which are part of the sensitivity concept. This warrants further examination for the role of OT in maternal sensitive caregiving behavior as well as maternal brain responses in a group of mothers whose maternal behavior has been rigorously defined.

Knowing the unique patterns of brain and OT activation that might be related to sensitive parenting, and using changes in brain activation in response to infant stimuli, present the possibility of identifying potential biomarkers. Such biomarkers may be used not only to evaluate new diagnostic and treatment strategies, but also to better target costly interventions in mothers with low maternal sensitivity.

In the current study, we aimed to chart distinct neurobiological profiles between 'higher' and 'lower' sensitivity mothers to test the following hypotheses: (i) in response to own versus unknown infant video, higher sensitivity mothers (HSM's) show increased BOLD activations compared to low sensitivity mothers (LSM's) in cortico-limbic circuits regulating motivation, reflexive caring, emotions and cognitions. Specifically, these include hippocampus, hypothalamus, cingulate cortex and STG); (ii) In HSM's, in response to own infant stimuli, BOLD activation correlates positively with maternal plasma OT responses following play interaction with their infants.

\section{Methods and Materials}

\section{Participants}


Between Feb 2011 and Oct 2011, 105 ethnically white British expectant mothers were recruited from six community antenatal clinics across Greater Manchester. Following informed consent, at 33.9 weeks $(\mathrm{SD}=3.19)$ antepartum, mothers were screened for mental illness using a clinical interview, Edinburgh Postnatal Depression Scale (EPDS), and Hospital Anxiety and Depression rating Scale (HADS). Mothers were excluded if they had mental illness or scored EPDS $\geq 12$ and/or HADS-depression $>11$. At 4-6 months (19.38 weeks, $\mathrm{SD}=2.47$ ) postpartum, 80 mothers were followed up and given behavioral assessment for maternal sensitivity through observed mother-infant dyads using the Manchester Assessment of Caregiver Infant Interaction (see below). Fourteen were lost to follow-up, 9 discontinued participation and 2 were no longer eligible ( 1 infant not living with mother; 1 infant death).

Of the 80 mother-infant dyads, 30 were selected based on lowest and highest maternal sensitivity rating and followed up at 7-9 months (35.14 weeks, $\mathrm{SD}=3.26)$. All mothers were right-handed, had no contraindication to MRI and were living with their infant. Of these mothers, 15 were rated as 'sensitive' and 15 as 'less sensitive'. Respectively, they were referred to as 'high sensitivity mothers' (HSMs) and 'low sensitivity mothers' (LSMs). The selection of the HSMs and LSMs were initially determined by taking ratings of 1+ SD above the mean (ratings 5-7), and 1+ SD below the mean (ratings 1-2), respectively, based on published MACI data on a healthy sample of 47 mothers at 7 months postpartum (Wan et al., 2012). However, as this did not provide, in the current sample, the required number of $\mathrm{N}=15$ in each group (based on power calculation for the scanning) due to the drop in the original sample at this phase, the thresholds were extended, but with no overlap, such that HSM's included those rated 4-7 (mean=4.47; $\mathrm{SD}=0.74)$ and LSM's included those rated 1-3 (mean=2.13; $\mathrm{SD}=0.52)$. The study protocol was approved by the North West Research Ethics Committee: Ref: 10/H1013/69. 


\section{Measures}

Manchester Assessment of Caregiver-Infant Interaction (MACI) (Wan et al., 2012, 2013, 2016)

This validated rating scale measure of global qualities of parent-infant interaction is suitable for 3-15 months of age and was designed to measure seven key areas on a 1 to 7 scale based on 6 minutes of videotaped unstructured play (Wan et al., 2016). A trained coder, who has undergone $>70$ hours training and supervised coding practice, rated the videotaped interactions following detailed reviews and narrative notes of the play episode, blind to family information and study aims. Each clip typically requires 30-40 minutes to rate. The measure has been used in normative and at-risk samples (Wan et al., 2012 \& 2013), in intervention contexts (Green et al., 2013 \& 2015) and in previous fMRI (Wan et al., 2014) and infant ERP research (Elsabbagh et al., 2014).

In the current study, maternal sensitivity was captured in the MACI 'caregiver sensitive responsiveness' scale. This is defined as the extent to which the infant's moment-to-moment behaviors are responded to and supported by the caregiver appropriately and contingently. Reliability was tested in two ways: (1) $30 \%$ of video clips were evaluated by a second independent trained and blinded coder and high inter-rater agreement was achieved (intraclass correlation: $\mathrm{r}=0.70 ; \mathrm{p}<0.001$; absolute agreement definition) and any disagreements were resolved by re-review and discussion to arrive at a consensus rating; (2) Parent-infant interactions were recorded again (for reliability purpose) during the second visit (when mothers attended for scanning and oxytocin measurement) and rated using the MACI by a third independent rater which achieved high consistency over time $(r=0.61 ; p<0.001)$, as we would expect as maternal sensitivity represents a relatively stable construct with some variability with infant and life changes. 
This 10-item self-report instrument is used to screen for depression in the postpartum and antenatal periods. Items are rated on a 4-point Likert scale. EPDS shows sensitivity from 68 to $95 \%$ and a specificity of $78 \%$ to $96 \%$ (Cox et al., 1987 ).

The Hospital Anxiety and Depression (HADS) rating scale (Zigmond\&Snaith,1983)

This 14-item questionnaire is a self-rating instrument to screen for anxiety and depression (7 items each). Items are rated on a 4-point Likert scale. The measure has high internal consistency and high test-retest reliability (Crawford et al., 2001).

\section{Procedure}

\section{Time 1: Mother-Infant Interaction}

Mothers were visited in their own homes at 4-6 months postpartum at a time when the infant was healthy and likely to be alert. After completing the informed consent, mothers were instructed to play with their infant for 6 minutes on a floor mat as they would normally do, with or without toys (supplied) as they prefer, ignoring the experimenter's presence. Interactions were videotaped by the same experimenter who meets the participant in all sessions of the study. Mothers were asked not to have visitors or other members of the family present during the interaction recording. Interactions were stopped and later resumed if the infant got distressed, sick or upon the mother's request. Mothers also completed EPDS, HADS and obstetric information sheet.

\section{Time 2: fMRI \& Oxytocin Sampling}

At 7-9 months postpartum, the 30 mothers attended the Wellcome Trust Clinical Research Facility in Manchester for OT blood sampling (before and after another interactive play) and fMRI scanning. 


\section{i. Oxytocin}

Three $5 \mathrm{ml}$ blood samples were taken from mothers (between 1200-1400 hours). Blood was taken from antecubital veins through an intravenous cannula. Upon arrival, mother and infant were separated for 10 minutes, after which the first sample (baseline) was taken. This was followed by reunion and a 10-minute mother-infant play interaction (as described earlier in Time 1). The second and the third sample were then taken immediately post interaction and 5 minute later respectively. The mean of the post-interaction samples (i.e. second and third samples) was taken to account for the pulsatile secretion of OT (Amico et al., 1985). To account for the physiological change of OT, breastfeeding mothers were asked to stop feeding one hour before their arrival.

Samples were drawn into chilled vacutainer tubes containing lithium heparin injected with $200 \mathrm{ml}$ of Trasylol (aprotinin) 500,000 KIU/ml blood. OT samples were kept ice-chilled and processed within 10 minutes. Samples were then centrifuged at $4^{\circ} \mathrm{C}$ at $3500 \mathrm{rpm}$ for 15 minutes. 500ul supernatants were transferred to two microtubes (aliquot $1 \& 2$ ) and stored at $80^{\circ} \mathrm{C}$ until transferred, on dry ice, to the University lab for analysis. OT was analysed using: ab13305-Oxytocin ELISA which is a valid and reliable method for measuring plasma OT levels (for protocol please refer to kithttp://www.abcam.com/oxytocin-elisa-kitab133050.html). Determination of OT was performed using the Max Binding Determination Competitive Assay protocol on Gen 5 software using a Biotek plate reader. Analyses include sample extraction (filtration of OT from its degradation products), which, according to Szeto et al. (2011), is necessary to obtain valid assay results through getting rid of multiple immunoreactive products that cast doubt on the specificity of the assays.

\section{ii. $\quad$ fMRI Paradigms}

For each infant, we produced a series of 20sec clips of 3 affects: (i) consistently smiling (happy) (ii) consistently crying (sad) and (iii) exhibiting no expression (neutral) for both the 
own and an 'unknown' infant (matched on age, gender) in addition to a neutral control stimulus (moving traffic) and rest (blank screen) (both 20sec). Videos were recorded with the infant sitting up, facing the camera, in front of a neutral background. Videos were matched for degree of movement of the infants and were presented without audio. Videos lasting 16 minutes were presented during scanning in the following order: (Own neutral - neutral control - unknown neutral infant) x 4, REST (i.e. blank screen), (Own happy - neutral control unknown happy infant) x 4, REST, (Own sad - neutral control - unknown sad infant) x 4, REST. All stimuli were displayed from a computer controlled projector presented on a display screen and relayed to the participant via a mirror placed above the head while in the MRI scanner. The task was temporally triggered by the ttl (transistor-transistor logic) pulse from the first slice acquisition of the MRI sequence.

\section{iii. fMRI Acquisition}

Imaging was performed using a1.5 Tesla Philips Intera MRI scanner running Explorer gradients (software version 11.1.4.4). High-resolution T1-weighted structural images were acquired to exclude any structural abnormality (none were found) and for co-registration with functional data. The structural scan, using 8 channel SENSE head coil, employed a 3D Contrast Turbo Field Echo Sequence with a temporal resolution (TR) of 9ms and an echo time (TE) of $4 \mathrm{~ms}$ with an $8^{\circ}$ flip angle producing 140 slices with a voxel size of $0.8 \times 0.8 \times$ $1.0 \mathrm{~mm}$. Functional images were acquired using a multi-slice, single-shot echo-planar imaging sequence, generating 29 ascending axial slices $(\mathrm{TR}=2.5 \mathrm{~s}, \mathrm{TE}=40 \mathrm{~ms}, 4 \mathrm{~mm}$ thickness with $0.5 \mathrm{~mm}$ slice gap, in-plane resolution of $3.4 \times 3.4 \mathrm{~mm}$ ).

iv. $\quad$ MRI Analysis

Imaging data were analysed using Statistical Parametric Mapping (SPM8 www.fil.ion.ucl.ac.uk/spm) implemented in MATLAB (MathworksInc, Sherborn, MA, USA) (Friston et al., 1996). Data were preprocessed, according to standard protocols; realigned 
using a least squares approach and a six-parameter (rigid body) spatial translation (Friston et al., 1996), spatially normalised onto standardised brain templates and smoothed using an isotropic Gaussian kernel filter (10mm full width half maximum [FWHM]). For statistical analysis, a parametric model was employed that enabled the modelling of linear hemodynamic responses. In order to quantify \%BOLD signal change in the paradigm, rest periods were regressed from video conditions (happy, sad and neutral own and unknown baby faces, traffic control) at the first level (Penny and Holmes, 2007). Second level statistical analyses compared brain activation patterns between our two maternal sensitivity groups using a two-factor random effects ANOVA, where differences in BOLD activation were assessed with a threshold of $\mathrm{p} \leq 0.05$ Family Wise Error (FWE), whole brain corrected for multiple comparisons.

To test our main hypotheses, we used a 'Region of Interest' (ROI) analysis with random effects comparing BOLD activations between the two groups of maternal sensitivity. A single ROI composite based on previous findings (Wan et al., 2014; Paul et al., submitted) was used to include 4 key areas involved in face emotion, attention and reward: right superior temporal gyrus, right posterior cingulate gyrus, left subthalamic nucleus and left hippocampal formation. The ROI was defined using anatomical boundaries (WFU PickAtlas (Maldjian et al, 2003) in SPM 8) and inference completed using a small volume correction for multiple comparisons $(\mathrm{p}<0.05 \mathrm{FWE})$. Activation was compared between the two groups of mothers, HSMs (N=15) and LSMs (N=15). Significant differences in the ROIs were further explored for correlation with plasma OT. The OT covariates were added to one sample t-tests of group differences and a small volume correction of the significant ROIs performed, corrected for multiple comparisons. 


\section{Results}

\section{Preliminary analysis: Background variables}

Maternal sensitivity ratings in this cohort appear largely to reflect the quality of the overall interaction (i.e. sensitivity scale correlated significantly with most other MACI scales). HSM and LSM groups did not differ in age, marital status, parental experience (primiparity), education, household income, mode of delivery or feeding, postpartum stage, infant birthweight, infant gender, or HADS anxiety and depression scores (Table 1). No relationship was found between current breastfeeding status and maternal sensitivity (Table 1). Plasma OT did not differ in breastfeeding mothers from other mothers either at baseline (mean=306.54 vs. $259.16, \mathrm{~F}(1,27)=1.78, \mathrm{p}=0.19)$ or post-interaction (mean=275.17 vs. $254.61, \mathrm{~F}(1$, 27) $=0.30, \mathrm{p}=0.59)$.

\section{Table 1about here please}

\section{Oxytocin results}

An outlier in plasma oxytocin ( $>3 \mathrm{SD}$ ) was excluded from all OT analyses. Strong correlations were found between baseline and post-interaction oxytocin levels in both HSMs ( $r=0.79$ 0.96; $\mathrm{p}<0.01)$ and LSMs $(\mathrm{r}=0.92-0.99 ; \mathrm{p}<0.01)$ supporting literature reports for high level of plasma OT individual stability (e.g. Levine et al., 2007). After excluding the outlier, household income was lower in LSMs than HSMs, and therefore was controlled for in the analyses. Baseline plasma OT was significantly lower among HSMs (mean=235.09; $\mathrm{SD}=83.51)$ than LSMs $(301.87 ; \mathrm{SD}=39.15), \mathrm{F}(1,26)=5.35 ; \mathrm{p}=0.03$. Similarly, postinteraction plasma OT was significantly lower in HSMs (mean=216.84; SD=79.18) than LSMs (mean=302.42; SD=36.27), F $(1,26)=9.77 ; \mathrm{p}<0.01)$ (see supplementary Figure, and for more details see Elmadih et al., 2014). 
Whole Brain Analyses in all mothers

Initially, we examined main effects using 'whole brain analyses' with a significant threshold $\mathrm{p} \leq 0.05$; Family Wise Error (FWE) corrected in all mothers combined. Comparing own versus unknown infant videos, significantly enhanced BOLD activation was observed in right IFG (BA $47 \&$ 9), and a range of subcortical regions, including left parahippocampal gyrus (BA 34), bilateral uncus (BA 28), all at $\mathrm{p}<0.05$ FWE, with borderline significant BOLD activation in anterior cingulate gyrus (BA, 24) (see Appendix).

\section{Comparisons between High and Low Sensitivity Mothers}

As mentioned earlier, this analysis was performed in a composite ROI comprising right superior temporal gyrus, right posterior cingulate gyrus, left subthalamic nucleus and left hippocampal formation. We compared responses to own versus unknown infant between maternal sensitivity groups for each facial affect separately [i.e. HSMs versus LSMs (own happy minus unknown happy infant)], HSMs versus LSMs (own neutral minus unknown neutral infant), HSMs versus LSMs (own sad minus unknown sad infant)], and similarly for LSMs versus HSMs. HSMs showed greater BOLD response, compared to LSMs, in right STG (BA 41) in response to own neutral as compared to unknown neutral infant (Table 2, Figure 1); whereas LSMs, compared to HSMs, did not show significant activation in response to own versus unknown infant contrasts in any of the ROIs. Comparing activation between HSMs and LSMs in own infant happy or sad versus unknown happy or sad videos did not reveal significant differences between groups.

Finally, we compared BOLD response to different facial affects between HSMs and LSMs using only own infant stimuli versus neutral control, [i.e. HSMs versus LSMs (own neutral infant minus neutral control), HSMs versus LSMs (own happy infant minus neutral control), HSMs versus LSMs (own sad infant minus neutral control)], and similarly for LSMs versus 
HSMs. Compared to LSMs, HSMs showed greater BOLD response in the right STG extending to right insula (BA 13) in response to own happy infant (minus neutral control) (Table 2, Figure 1). By contrast, compared to HSMs, LSMs did not show significant BOLD activation in response to own happy infant video in any ROIs. Viewing own neutral or sad infant videos (each minus neutral control) did not reveal significant differences in BOLD response in the ROIs when the two groups were compared.

\section{Table 2 about here please}

\section{Figure 1 about here please}

\section{Correlations of Plasma Oxytocin with ROI Activation}

We examined ROI response against post-interaction maternal plasma OT and found activation of right STG (in response to own happy infant versus neutral control) was negatively correlated with plasma OT levels among higher sensitivity mothers ( $r=-0.81$; $\mathrm{p}<0.01$ ) (Figure 2). BOLD activation in response to own neutral as compared to unknown neutral infant was not correlated with plasma OT. On the other hand, in LSMs there was no significant correlation between post-interaction plasma OT and any of the a priori ROIs.

\section{Figure 2 about here please}

\section{Discussion}

When healthy postpartum mothers viewed videos of their own compared to unknown infants, significant brain response was elicited predominantly in right inferior frontal gyrus, left parahippocampal gyrus and bilateral uncus, and anterior cingulate gyrus (borderline). This is consistent with previous findings using baby cry and visual stimuli (Atzil et al., 2012; Ho et 
al., 2014; Kim et al., 2011; Phelps, 2004; Swain et al., 2008; Wittfoth-Schardt et al., 2012). This activation pattern may reflect the role of inferior frontal gyrus and anterior cingulate gyrus in the decoding of facial expressions of emotions (Adolphs, 2002; Barrett \& Fleming, 2011). A key to healthy maternal parenting is the capacity of mother to recognise and respond to her infant's emotions (Strathearn et al., 2012). Activation of parahippocampal gyrus and uncus supports previous evidence that these regions are particularly important in encoding emotional memories (Phelps, 2004).

When compared to LSMs, HSMs showed greater BOLD response in right STG, in response to viewing videos of own compared to an unknown infant in neutral condition and also to own happy infant compared to neutral control. When compared to HSMs, LSMs showed no significantly greater response in any ROI in response to their own infants. However, inconsistent with our second hypothesis, the BOLD response in the right STG among HSMs in response to own happy infant (versus neutral control) was negatively, not positively, correlated with maternal post-play interaction plasma OT concentrations.

Enhanced activation of STG to videos of own versus unknown infants has also been reported in a recent similar study (Wan et al., 2014); and in a study of synchronous (cfd. intrusive)mothers (Atzil et al., 2011). This region has been widely implicated in the regulation of emotion, particularly facial emotion processing, and in empathising with others 'Theory of Mind' (Rizzolatti \& Fabbri-Destro, 2008) - both facilities are key for a mother to differentially express sensitivity to her infant's needs over other calls on her resources (Strathearn et al., 2012). Although not direct measures of variation in maternal sensitivity, other previous studies have also reported greater activation of STG in mothers who delivered vaginally compared to by Caesarean section (Swain et al., 2008) and in breastfeeding compared to bottle feeding mothers (Kim et al., 2011). 
Developmental theories suggest that sensitive mothers must have the capacity to recognise her infant's emotional and other cues/signals, differentially pay attention to them and then respond to them by appropriately mirroring and affirming positive emotions or recognising and reassuring in relation to negative ones (Ainsworth et al., 1978; Sroufe, 2000). The pattern of brain response our HSMs show (compared to LSMs) might be related to superior maternal reflective capacity (Brunet et al., 2000; Lenzi et al. 2009) and imply greater attentional attribution of those mothers towards their own infant. HSMs and LSMs' were only significantly different in their response to neutral emotion and not to happy or sad emotion. Neutral infant cues may demand more attention as the mother tries to interpret the nature of the infant's expression; whereas happy and sad infant faces may be more readily interpretable.

Prior studies considered maternal sensitivity to be the mother's response to the infant's mental state, rather than to her/his physical state (Fonagy et al., 1994; Meins, 2001); thus a sensitive mother would be expected to respond appropriately to her infant's displayed emotions by affirming the positive emotions and/or reassuring them about the negative ones (Sroufe, 2000).

Here, we report how HSMs did not show significantly greater BOLD response than LSMs in regions involved in OT regulation or motivation e.g. respectively the thalamus and posterior cingulate gyrus. Previous findings are equivocal, with some studies reporting greater activation of these areas in response to infant cry (Kim et al., 2011; Lorberbaumet al., 2002; Swain et al., 2004; Swain et al., 2008), while others did not (Bartels \&Zekiet al., 2004; Seifritzet al., 2003). Our findings among all mothers (main effect) may reflect aspects of maternal functioning other than the mother's appropriate and prompt response to her infant. The areas highlighted here may be important for aspects of maternal sensitivity that overlap more with related social behaviors: for example, anterior cingulate, known to be important in 
social reward (Bolling et al., 2011; Ho et al., 2012) and self-referential processing of emotional stimuli (Somerville et al., 2010; Yoshimura et al., 2014).

Contrary to our expectations, we found that the BOLD response in right STG among HSMs in response to own happy infant (versus neutral control) was negatively, not positively correlated with their post-play plasma OT levels. While these findings are contrary to some recent parenting studies which reported positive correlations between plasma OT levels and positive maternal (or paternal) behaviors (e.g. Feldman et al., 2010) or brain responses in synchronous mothers (Atzil et al., 2011), they are in line with other studies that implicate OT's importance in regulating interpersonal stress in women (Tabak et al., 2011; Taylor et al., 2006, 2010) including mothers (Feldman et al., 2011; Strathearn et al., 2012). It is also important to note that this is only the second study to have examined maternal brain responses in relation to plasma OT alongside maternal behavior, and the first to consider grouping mothers based on maternal sensitivity rating.

Strathearn et al (2012) investigated the relationship between adult temperament and plasma OT responses among 55 first time mothers and their 6-7 month-old infants. They reported that maternal plasma OT responses were negatively correlated with maternal effortful control, i.e. the propensity to focus on executing plans, performing tasks and maintaining focus and attention. In other words, the more concentration and attention shown by the mother during interaction with her infant, the less likely she was to experience an increase in plasma OT following the interaction. Characteristics related to effortful control are important for sensitive mothering as mothers need focus attention to respond 'promptly' and 'appropriately' to infant signals (Ainsworth et al., 1978). Another group reported a significant drop in plasma OT in 32 nulliparous women in response to laboratory-induced positive emotion (i.e. a comedy movie), whereas no change was found after women viewed negative emotions (sad movie) (Turner et al., 2002). Bick \& Dozier (2010) measured urinary OT levels (known to 
correlate significantly with plasma level- Hoffman et al., 2012) in 26 healthy mothers following play-interactions with their child and again with an unfamiliar child. Urinary OT was significantly higher following interaction with the unfamiliar child as compared with own child. They concluded that interaction with an unfamiliar child might constitute a (socially) stressful affiliative situation and that OT might have increased in an attempt to modulate this stress. Our findings in relation to OT support the notion of simultaneous, multiple roles for OT both reflecting and facilitating affiliative relationships as suggested by Numan \& Woodside, 2010 and others (Taylor et al., 2010). This notion is supported by a number of other recent findings (Swain et al., 2014a).

Thus, significantly higher urinary OT has also been reported following interaction with own child in mothers who experienced gaps in positive social relationships (with her own mother or romantic partner);in mothers who experienced infant's negative engagement; and in those who showed interactive stress (i.e. proportion of time when the infant shows negative reactivity while the mother tries to re-engage her/him during 'observed' interactive play) (Feldman et al., 2011). Interestingly, in Feldman et al's study, higher baseline plasma OT and post-interaction salivary OT levels were reported among parents with high affective synchrony. The absence of correlation between plasma/salivary OT and urinary OT in the study by Feldman et al., might suggest there are indeed simultaneous and multiple roles for the OT. In a separate, but related experiment (Elmadih et al., 2014), we have examined mothers' own experience of parenting using the Parental Bonding Instrument (Parker et al., 1979). Among low sensitivity mothers only, both baseline and post interaction plasma OT levels correlated significantly and positively with perceived maternal overprotection. Therefore, plasma OT elevation among LSMs in the current study could be a reflection of their negative recall of their own maternal parenting experience (as a representation for a difficult relationship with own mother). 
Evidence is now accumulating that, at least in women, OT is triggered/released when there is a need to enhance or promote affiliation with others or when the social relationship is 'threatened' (including pair bonding or parental bonding) (Taylor et al., 2006, 2010; Bick \& Dozier, 2010; Feldman et al., 2011). Women may use their close relationships with others in a different way to men: women may regulate their perception and responses to stress through the development of close affiliative bonds (Ho et al., 2014; Marazziti et al., 2006). In this way, plasma OT may be secreted in some women particularly under what they perceive to be stressful situations in order specifically to prompt a desire for affiliation (Taylor et al., 2006). Interestingly, this dual action for OT, as a pro-social, pro-affiliative as well as an anti-stress hormone might be represented simultaneously within the same individual, as suggested by Feldman et al. (2011).

It is possible that our data support a role for OT in reducing or modifying social stress (Taylor et al., 2010). Specifically, compared to LSMs, mothers at the higher end of the sensitivity distribution (i.e. HSMs) may simply perceive their infants, and infant interaction, as more positive and give it more effortless concentration and attention as evidenced by their lower plasma OT levels following own infant interaction. In line with this, HSM mothers also activated brain regions that indicate a greater ability to recognise and understand their infant's emotional cues (STG) (Iacoboni \& Dapretto, 2006).

\section{Strength and Limitations}

As far as we are aware, this is the first study to chart differences in neural responses, as well as plasma OT responses, in mothers with contrasting degrees of sensitive infant caregiving (LSMs=minimal to scattered sensitivity; HSMs=mixed to high sensitivity).Our two groups of mothers were selected from a relatively large maternal sensitivity cohort . Participants were screened for depression and excluded from the study if they showed depressive symptoms to 
minimize the influence of maternal mood on brain/OT responses. There remain some important limitations. First, inferences about central OT from plasma measurements must remain limited (Kagerbauer et al., 2013) despite reported modulation of peripheral plasma OT in relation to social affiliation (e.g. Atzil et al., 2011; Strathearn et al., 2009; 2012). Secondly, selection of the HSMs and LSMs was initially determined by taking ratings of $1+$ above the SD (ratings 5-7), and 1+ below the SD (ratings 1-2) (Wan et al., 2012). However, as this did not provide, the required number of 15 in each group the thresholds were increased, but with no overlap. Only 3 of those rated 4 were included in HSMs group and only 2 of those rated 3 were included in LSMs group, the rest were within the ranges which were set initially and the two groups still showed distinct neurobiological profiles. Thirdly, the significant correlation between plasma OT and BOLD activation among HSMs does not imply a causal relationship; other unidentified factors (e.g. relationship with partner) might also account for this. Finally, we confined our recruitment to white women (as a requirement for other parts of the study); this may limit the generaliseability of our findings to other groups.

\section{Conclusion}

This study brings together physiological, neurophysiological and behavioral aspects of human parenting. Originating from a healthy, population-based sample recruited antenatally, our findings suggest that particular areas of brain associated with emotion processing are activated in mothers when they view videos of their own infant. STG is activated during facial emotion perception; our findings implicate the STG, in particular, in distinguishing more highly sensitive mothers. Although OT is known for its affiliative role, our findings suggest that the greater capacity for facial emotion processing (greater STG activation)shown by HSMs in response to own infant stimuli, is associated with lower plasma OT. In contrast, lower maternal sensitivity, unaccompanied by BOLD activation in response to infant stimuli, 
is accompanied by higher levels of plasma OT levels both at baseline and following play with own infant. These higher levels of plasma OT correlate positively with negative recall of own maternal parenting experience (higher maternal overprotection) in those mothers.

Future research should examine how STG activation and/or dynamic OT challenge may respond following parenting interventions demonstrated to improve maternal sensitivity (Riem et al., 2011; Swain et al., 2012, 2014b). Such biomarkers may be valuable in identifying which mothers with behavioral evidence of poor sensitivity should be targeted for intervention, as well as monitoring likely improvements in such mother's longer term. This might be of great importance considering the difficulty in assessing 'efficiency of interventions' (Bakermans-Kranenburg\& Van Ijzendoorn, 2015). This strategy could also provide for more efficient, randomized controlled trial designs. Research might also consider the simultaneous assessment of maternal urinary or salivary OT along with plasma OT measures. Previous evidence suggests that elevation of plasma OT in relation to stress is only in the context of stimuli which are social stressors i.e. social relationship difficulties. However, future studies replicating the present design might usefully combine measurements of plasma OT with additional measures to evaluate the HPA or stress axis (e.g. cortisol) for more thorough evaluation of the role of OT in stress regulation (Quirin et al., 2011).

\section{Acknowledgements}

This study was supported by a Magnetic Resonance Imaging Facility grant, the Wellcome Trust Clinical Research Facility (Manchester), and the Centre for Women's Mental Health (University of Manchester, UK). Authors declare no conflict of interest. 


\section{References}

Adolphs R (2002): Neural systems for recognizing emotion. Current Opinion in Neurobiology 12, 169-77.

Ainsworth MDS, Blehar MC, Waters E \& Wall S (1978): Patterns of attachment: A psychological study of the strange situation. Hillsdale, NJ: Erlbaum.

Alink LRA, Mesman J, Van Zeijl J, Stolk MN, Juffer F, Bakermans-Kranenburg MJ, Van IJzendoorn MH \&Koot HM(2008): Maternal Sensitivity Moderates the Relation between Negative Discipline and Aggression in Early Childhood. Parenting and Early Aggression 18, 99-120.

Amico JA, Ervin MG, Leake RD, Fisher DA, Finn FM \& Robinson AG (1985): A novel oxytocin-like and vasotocin-like peptide in human plasma after administration of estrogen. Journal of clinical endocrinology and metablolism60, 5-12.

Atzil S, Hendler T \& Feldman R (2011): Specifying the Neurobiological Basis of Human Attachment: Brain, Hormones, and Behavior in Synchronous and Intrusive Mothers. Neuropsychopharmacology36, 1-13.

Atzil S, Hendler T, Zagoory-Sharon O, Winetraub Y \& Feldman R (2012): Synchrony and specificity in the maternal and the paternal brain: relations to oxytocin and vasopressin. Journal of the American Academy of Child and Adolescent Psychiatry 51, 798-811.

Bakermans-Kranenburg MJ\& Van IJzendoorn MH (2015): The hidden efficacy of interventions: Gene x Environment experiments from a differential susceptibility perspective. Annual Review of Psychology, 66, 381-409.

Barlow J, Schrader McMillan A\& Kirkpatrick S (2008): Health-led parenting interventions in pregnancy and early years. Research Report DCSF-RW070.9 
Barrett J \& Fleming AS (2011): Annual Research Review: All mothers are not created equal: Neural and psychobiological perspectives on mothering and the importance of individual differences. Journal of Child Psychology and Psychiatry52, 368-97.

Bartels A\&Zeki S (2004): The Neural correlation of maternal and romantic love.

Neuroimage21, 1155-66.

Bick J \& Dozier M (2010): Mothers' and Children's Concentrations of Oxytocin Following Close, Physical Interactions with Biological and Non-biological Children. Developmental Psychobiology52, 100-7.

Bigelow AE, Bigelow AE, MacLean K, Proctor J, Myatt T, Gillis R \& Power M(2010): Maternal sensitivity throughout infancy: continuity and relation to attachment security. Infant Behavior and Development 33, 50-60.

Bolling DZ, Pitskel NB, Deen B, Crowley MJ, McPartland JC, Mayes LC \&Pelphrey KA (2011): Dissociable brain mechanisms for processing social exclusion and rule violation. Neuroimage54, 2462-71.

Brunet E, Sarfati Y, Hardy-Bayle MC \&Decety J (2000): A PET Investigation of the Attribution of Intentions with a Nonverbal Task. Neuroimage11, 157-166.

Cox JL, Holden JM \&Sagovsky R (1987): Detection of postnatal depression: Development of the 10-item Edinburgh Postnatal Depression Scale. British Journal of Psychiatry150, $782-786$.

Crawford JR, Henry JD, Crombie C \& Taylor EP (2001): Normative data for the HADS from a large non-clinical sample. British Journal of Clinical Psychology 40, 429-34.

Crosnoe R, Leventhal T, Wirth RJ, Pierce KM \&Pianta RC; NICHD Early Child Care Research Network. (2010): Family socioeconomic status and consistent environmental stimulation in early childhood. Child Development81, 972-87. 
Downer JT \&Pianta RC (2006): Academic and cognitive functioning in first grade: associations with earlier home and child care predictors and with concurrent home and classroom experiences. School Psychology Review35, 11-30.

Elmadih A, Wan MW, Numan M, Elliott R, Downey D \& Abel KM (2014). Does oxytocin modulate variation in maternal caregiving in healthy new mothers? Brain Research1580, 143-150.

Elsabbagh M, Bruno R, Wan MW, Charman T, Johnson MH \& Green J(2014): Infant neural sensitivity to dynamic eye gaze relates to quality of parent-infant interaction at 7Months in infants at risk for autism. Journal of Autism and Developmental Disorders, $1-9$.

Feldman R, Weller A, Zagoory-Sharon O \& Levine A (2007). Evidence for a neuroendocrinological foundation of human affiliation: plasma oxytocin levels across pregnancy and the postpartum period predict mother-infant bonding. Psychological Science18, 965-70.

Feldman, R., Gordon, I. \&Zagoory-Sharon, O. (2010). The cross-generation transmission of oxytocin in humans Hormones and Behavior58, 669-76.

Feldman R, Gordon I \&Zagoory-Sharon O (2011): Maternal and paternal plasma, salivary, and urinary oxytocin and parent-infant synchrony: considering stress and affiliation components of human bonding. Developmental Science14, 752-61.

Fonagy P, Steele M, Steele H, Higgitt A \& Target M (1994): 'The Emanuel Miller Memorial Lecture 1992'. The Theory and Practice of Resilience.Journal of Child Psychology and Psychiatry35, 231-57.

Friston KJ, Holmes A, Poline JB, Price CJ \&Frith CD (1996): Detecting activations in PET and fMRI: levels of inference and power. Neuroimage4, 223-35. 
Gordon I, Zagoory-Sharon O, Leckman JF \& Feldman R (2010): Oxytocin and the Development of Parenting in Humans. Bilological Psychiatry 68, 377-82.

Green J, Wan MW, Guiraud J, Holsgrove S, McNally J, Slomins V, Elsabbagh M, Charman T, Pickles A, Johnson M \& The BASIS Team (2013): Intervention for infants at high risk of developing autism - A case series. Journal of Autism and Developmental Disorders, 43, 2502-2514.

Green J, Charman T, Pickles A, Wan MW, Elsabbagh M, Slonims V, Taylor C, McNally J, Booth R, Gilga T, Jones E, Harrop C, Bedford R, \& Johnson MH (2015): Parentmediated intervention for infants at high risk of autism: Randomised clinical trial. Lancet Psychiatry, 2, 133-140.

Hoffman ER, Brownley KA, Hamer RM \&Bulik CM: (2012). Plasma, salivary, and urinary oxytocin in anorexia nervosa: a pilot study. Eating Behaviors 13, 256-9.

Ho SS, Gonzalez RD, Abelson JL, \&Liberzon I (2012): Neurocircuits underlying cognitionemotion interaction in a social decision making context. Neuroimage, 63, 843-857.

Ho SS, Konrath S, Brown SL \& Swain JE (2014): Empathy and Stress Related Neural Responses in Maternal Decision Making. Fronties in Neuroscience.8, 152.doi: 10.3389/fnins.2014.00152

Iacoboni M \&Dapretto M (2006): The mirror neuron system and the consequences of its dysfunction. Nature Reviews Neuroscience7, 942-51.

Kagerbauer SM, Martin J, Schuster T, Blobner M, Kochs EF, Landgraf R (2013): Plasma oxytocin and vasopressin do not predict neuropeptide concentrations in human cerebrospinal fluid. Journal of Neuroendocrinology25, 668-673. 
Kim P, Feldman R, Mayes LC, Eicher V, Thompson N, Leckman JF \& Swain JE (2011): Breastfeeding, brain activation to own infant cry, and maternal sensitivity. Journal of Child Psychology and Psychiatry 52, 907-15.

Kochanska G \& Kim S (2012): Difficult temperament moderates links between maternal responsiveness and children's compliance and behavior problems in low-income families. Journal of Child Psychology and Psychiatry 54, 323-32.

Lee HJ, Macbeth AH, Pagani JH \& Young WS (2009): Oxytocin: the great facilitator of life. Progress in Neurobiology.88, 127-51.

Lenzi D, Trentini C, Pantano P, Macaluso E, Iacoboni M, Lenzi GL \&Ammaniti M (2009): Neural basis of maternal communication and emotional expression processing during infant preverbal stage. Cerebral Cortex19, 1124-33.

Levine A, Zagoory-Sharon O, Feldman R \& Weller A (2007): Oxytocin during pregnancy and early postpartum: Individual patterns and maternal-fetal attachment Peptides 28, 1162-69.

Lorberbaum JP, Newman JD, Horwitz AR, Dubno JR, Lydiard RB, Hamner MB, et al. (2002): A potential role for thalamocingulate circuitry in human maternal behavior. Biological Psychiatry51, 431-45.

Maldjian JA, Laurienti PJ, Kraft RA, Burdette JH (2003): An automated method for neuroanatomic and cytoarchitectonic atlas-based interrogation of fMRI data sets. Neuroimage19, 1233-1239.

Marazziti D, Dell'Osso B, Baroni S, Mungai F, Catena M, Rucci P, Albanese F, Giannaccini G, Betti L, Fabbrini L, Italiani P, Del Debbio P, Lucacchini A \&Dell'Osso L (2006): A relationship between oxytocin and anxiety of romantic attachment. Clinical Practice and Epidemiology in Mental Health 28. 
Meins EAF, Fradley E \& Tuckey M (2001): Rethinking maternal sensitivity: mothers' comments on infants' mental processes predict security of attachment at 12 months. Journal of child psychology and psychiatry42, 637-648.

Musser ED, Kaiser-Laurent H \& Ablow JC (2012): The neural correlates of maternal sensitivity: an fMRI study. Developmental Cognitive Neuroscience 2, 428-36.

Noriuchi M, Kikuchi Y \&Senoo A (2008): The functional neuroanatomy of maternal love: mother's response to infant's attachment behaviors. Biological Psychiatry 63, 415-23.

Numan M \& Woodside B (2010): Maternity: neural mechanisms, motivational processes, and physiological adaptations. Behavioral Neuroscience124, 715-41.

Parker G, Tupling H, and Brown LB (1979): Parental bonding instrument. British Journal of Medical Psychology 52, 1-10.

Pearson RM, Heron J, Melotti R, Joinson C, Stein A, Ramchandani PG \& Evans J (2011):

The association between observed non-verbal maternal responses at 12 months and later infant development at 18 months and IQ at 4 years: a longitudinal study. Infant Behavior and Development 34, 525-33.

Penny WD and Holmes AJ (2007): Random-Effects Analysis. In Human Brain Function, Frackowiak RS, Friston KJ, Frith CD, Dolan RJ, Price CJ, Zeki S, Ashburner J, and Penny WD, eds. (San Diego: Elsevier Academic Press), pp. 156-165.

Phelps EA (2004): Human emotion and memory: interactions of the amygdala and hippocampal complex. Current Opinion in Neurobiology 14, 198-202.

Quirin M, Kuhl J \& Dusing R (2011): Oxytocin buffers cortisol responses to stress in individuals with impaired emotion regulation abilities. Psychoneuroendocrinology 36, 898-904.

Ranote S, Elliott R, Abel KM, Mitchell R, Deakin JF \& Appleby L 2004): The neural basis of maternal responsiveness to infants: an fMRI study. Neuroreport15, 1825-29. 
Riem MM, Bakermans-Kranenburg MJ, Pieper S, Tops M, Boksem MA, Vermeiren RR, et al. (2011): Oxytocin modulates amygdala, insula, and IFG responses to infant crying: a randomized controlled trial. Biological Psychiatry 70, 291-7.

Rizzolatti G \&Fabbri-Destro M (2008): The mirror system and its role in social cognition. Current Opinion in Neurobiology 18, 179-84.

Sandler I, Schoenfelder E, Wolchik S \& MacKinnon D (2011): Long-term impact of prevention programs to promote effective parenting: Lasting effects but uncertain processes. Annual review of psychology, 62, 299.

Seifritz E, Esposito F, Neuhoff JG, Luthi A, Mustovic H, Dammann G, et al. (2003): Differential sex-independent amygdala response to infant crying and laughing in parents versus nonparents. Biological Psychiatry54, 1367-75.

Somerville LH, Kelley WM \& Heatherton TF (2010): Self-esteem Modulates Medial Prefrontal Cortical Responses to Evaluative Social Feedback. Cerebral Cortex20, 300513.

Sroufe L (2000): Early relationships and the development of children. Infant Mental Health Journal21, 67-74.

Strathearn L, Fonagy P, Amico J \& Montague PR (2009): Adult attachment predicts maternal brain and oxytocin response to infant cues. Neuropsychopharmacology34, 2655-66.

Strathearn L, Iyengar U, Fonagy P \& Kim S (2012): Maternal oxytocin response during mother-infant interaction: Associations with adult temperament. Hormones and Behavior 61, 429-35.

Swain JE, Leckman JF, Mayes LC, Feldman R, Constable RT \& Schultz RT (2004): Neural substrates and psychology of human parent-infant attachment in the postpartum. Biological Psychiatry55. 
Swain JE, Tasgin E, Mayes LC, Feldman R, Constable RT \&Leckman JF (2008): Maternal brain response to own baby-cry is affected by Cesarean section delivery. Journal of Child Psychology and Psychiatry 49, 1042-52.

Swain JE, Konrath S, Brown SL, Finegood ED, Akce LB, Dayton CJ \& Ho SS (2012): Parenting and Beyond: Common Neurocircuits Underlying Parental and Altruistic Caregiving. Parenting, Science and Practice12, 115-23.

Swain JE, Kim P, Spicer J, Ho SS, Dayton CJ, Elmadih A \& Abel KM (2014a): Approaching The Biology Of Human Parental Attachment: Brain Imaging, Oxytocin And Coordinated Assessments Of Mothers And Fathers. Brain Research.doi:

\subsection{6/j.brainres.2014.03.007.}

Swain JE, Ho SS, Dayton CJ, Rosenblum KL, \&Muzik M (2014b): Attachment Intervention for Trauma-Exposed Mothers Affects Stress and Empathy Neurocircuits. Paper presented at the 34th Annual Meeting of the Canadian Academy of Child and Adolescent Psychiatry, Toronto.

Szeto A, McCabe PM, Nation DA, Tabak BA, Rossetti MA, McCullough ME, Scheiderman N, Mendez AJ, 2011: Evaluation of enzyme immunoassay and radioimmunoassay methods for the measurement of plasma oxytocin. Psychosomatic Medicine 73, 393-400.

Tabak BA, McCullough ME, Szeto A, Mendez AJ \& McCabe PM (2011). Oxytocin indexes relational distress following interpersonal harms in women. Psychoneuroendocrinology36, 115-22.

Taylor SE, Gonzaga GC, Klein LC, Hu P, Greendale GA \&Seeman SE (2006): Relation of oxytocin to psychological stress responses and hypothalamic-pituitary-adrenocortical axis activity in older women. Psychosomatic Medicine68, 238-45. 
Taylor SE, Saphire-Bernstein S \&Seeman TE (2010): Are plasma oxytocin in women and plasma vasopressin in men biomarkers of distressed pair bond relationships? Psychological Science21, 3-7.

Turner RA, Altemus M, Yip DN, Kupferman E, Fletcher D, Bostrom A, Lyons DM \&Amico JA (2002): Effects of emotion on oxytocin, prolactin, and ACTH in women. Stress5, 269-76.

Wan MW, Green J, Elsabbagh M, Johnson M, Charman T \& Plummer F; BASIS Team (2012): Parent-infant interaction in infant siblings at risk of autism. Research in Developmental Disabilities 33, 924-32.

Wan MW, Green J, Elsabbagh M, Johnson M, Charman T, Plummer F; the BASIS Team (2013): Quality of interaction between at-risk infants and caregiver at $12-15$ months is associated with 3-year autism outcome. J Child Psychol Psychiatry7, 763-71.

Wan MW, Downey D, Strachan H, Elliott R, Williams SR \& Abel KM (2014): The neural basis of maternal bonding. PLoS One, 9(3): e88436.

Wan MW, Brooks A, Green J, Abel K \& Elmadih A (2016): Psychometrics and validation of a brief rating measure of parent-infant interaction: Manchester Assessment of Caregiver-Infant Interaction. International Journal of Behavioral Development.eScholarID: $\underline{300145}$

Warren SL \&Simmens SJ (2005): Predicting toddler anxiety, depressive symptoms: Effects of caregiver sensitivity on temperamentally vulnerable children. Infant Mental Health Journal26, 40-55.

Wittfoth-Schardt D, Gru“ nding J, Wittfoth M, Lanfermann H, Heinrichs M, Domes G, et al. (2012): Oxytocin modulates neural reactivity to children's faces as a function of social salience. Neuropsychopharmacology37, 1799-1807. 
Yoshimura S, Okamoto Y, Onoda K, Matsunaga M, Okada G, Kunisato Y, et al. (2014): Cognitive behavioral therapy for depression changes medial prefrontal and ventral anterior cingulate cortex activity associated with self-referential processing. Social Cognitive and Affective Neuroscience 9, 487-93.

Zigmond AS \&Snaith RP (1983): The hospital anxiety and depression scale. ActaPsychiatricaScandinavica67, 361-70. 
Table 1. The demographic and obstetric characteristics of mothers grouped by level of maternal sensitivity (high sensitivity mothers - HSMs, and low sensitivity mothers - LSMs)

\begin{tabular}{|c|c|c|c|c|c|}
\hline Characteristic & $\begin{array}{l}\text { HSMs } \\
(\mathrm{N}=15)\end{array}$ & $\begin{array}{l}\text { LSMs } \\
(\mathrm{N}=15)\end{array}$ & $\begin{array}{l}\text { Statistics } \\
\mathrm{t}(28)\end{array}$ & $\begin{array}{l}\text { Chi- } \\
\text { square } \\
\text { test }\end{array}$ & $\begin{array}{l}\mathrm{p} \text { - } \\
\text { value }\end{array}$ \\
\hline \multicolumn{6}{|l|}{ Mean [SD] } \\
\hline Maternal age (years) & $30.40[5.37]$ & $27.65[4.76]$ & -1.38 & & 0.18 \\
\hline $\begin{array}{l}\text { Average maternal } \\
\text { education (years) }\end{array}$ & $15.06[2.82]$ & $12.80[2.73]$ & -1.87 & & 0.07 \\
\hline $\begin{array}{l}\text { Average annual } \\
\text { household income } \\
\text { (thousand pounds) }\end{array}$ & $33.00[4.61]$ & $25.33[4.24]$ & -2.76 & & 0.09 \\
\hline $\begin{array}{l}\text { Infant birthweight } \\
\text { (kilograms) }\end{array}$ & $3.44[0.44]$ & $3.23[0.59]$ & -0.80 & & 0.43 \\
\hline $\begin{array}{l}\text { Postpartum stage } \\
\text { (weeks) }\end{array}$ & $35.93[2.81]$ & $34.29[3.69]$ & -1.37 & & 0.18 \\
\hline $\begin{array}{l}\text { HADS anxiety } \\
\text { scores }\end{array}$ & $5.07[3.73]$ & $4.33[2.23]$ & 2.08 & & 0.16 \\
\hline $\begin{array}{l}\text { HADS depression } \\
\text { scores }\end{array}$ & $1.93[2.05]$ & $2.07[1.58]$ & 0.46 & & 0.50 \\
\hline \multicolumn{6}{|l|}{ Frequency (\%) } \\
\hline Married/cohabiting & $13(86.7)$ & $11(78.6)$ & & 0.56 & 0.65 \\
\hline Primiparous & $6(40.0)$ & $9(64.3)$ & & 1.71 & 0.19 \\
\hline $\begin{array}{l}\text { Infant gender } \\
\text { (female) }\end{array}$ & $10(66.7)$ & $7(50.0)$ & & 0.83 & 0.36 \\
\hline $\begin{array}{l}\text { Mode of delivery } \\
\text { (vaginal) }\end{array}$ & $10(66.7)$ & $11(78.6)$ & & 0.51 & 0.47 \\
\hline $\begin{array}{l}\text { Mode of feeding } \\
\text { (breast) }\end{array}$ & $3(20.0)$ & $3(20.0)$ & & $0.55^{\mathrm{a}}$ & 0.64 \\
\hline
\end{tabular}

${ }^{\mathrm{a}}$ Fisher exact test 
Table 2. Areas of significant BOLD activation within ROI in response to infant stimuli, when comparing high sensitivity $(\mathrm{N}=15)$ and low sensitivity $(\mathrm{N}=15)$ mothers.

\begin{tabular}{|c|c|c|c|c|c|c|c|c|}
\hline \multirow[t]{2}{*}{$\begin{array}{l}\text { Groups } \\
\text { compared }\end{array}$} & \multirow[t]{2}{*}{ Contrast } & \multirow[t]{2}{*}{ ROI } & \multirow[t]{2}{*}{$\mathbf{B A}$} & \multicolumn{3}{|c|}{$\begin{array}{l}\text { Talairach } \\
\text { coordinates } \\
\end{array}$} & \multirow[t]{2}{*}{$\begin{array}{l}\mathrm{Z} \\
\text { score }\end{array}$} & \multirow[t]{2}{*}{ FWE } \\
\hline & & & & $\mathbf{x}$ & $\mathbf{y}$ & $\mathbf{z}$ & & \\
\hline \multirow{3}{*}{$\begin{array}{l}\text { High } \\
\text { sensitivity } \\
\text { mothers vs. } \\
\text { Low } \\
\text { sensitivity } \\
\text { mothers }\end{array}$} & $\begin{array}{l}\text { Own neutral } \\
\text { infant minus } \\
\text { unknown neutral } \\
\text { infant }\end{array}$ & $\begin{array}{l}\text { Superior } \\
\text { temporal } \\
\text { gyrus }\end{array}$ & 41 & 57 & -25 & 13 & 3.39 & 0.05 \\
\hline & Own happy & Superior & 41 & 43 & -32 & 5 & 3.92 & 0.01 \\
\hline & $\begin{array}{l}\text { infant minus } \\
\text { neutral control }\end{array}$ & $\begin{array}{l}\text { temporal } \\
\text { gyrus }\end{array}$ & 13 & 57 & -32 & 18 & 3.38 & 0.05 \\
\hline
\end{tabular}

Note. All significant ROI (Family Wise Error $(F W E) \leq 0.05)$ corrected for multiple comparison. BA: Brodmann's area; L: Left, R: Right. 


\section{Figure 1.}
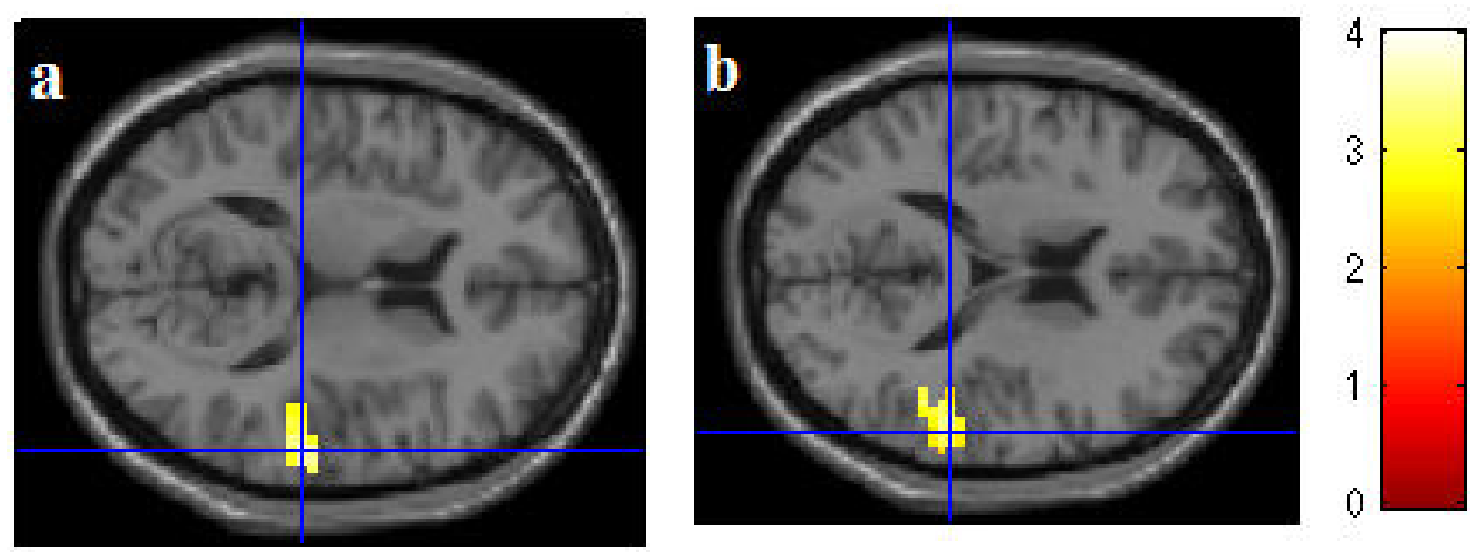

Figure 1. Maternal brain activation in response to infant stimuli: Compared with LSMs, HSMs show greater activation of the right superior temporal gyrus in response to: (a) own neutral infant versus unknown neutral infant videos, and (b) own happy infant versus neutral control, at ROI-threshold of FWE $\leq 0.05$. Structural brain image created from average of all subjects. 
Figure 2.

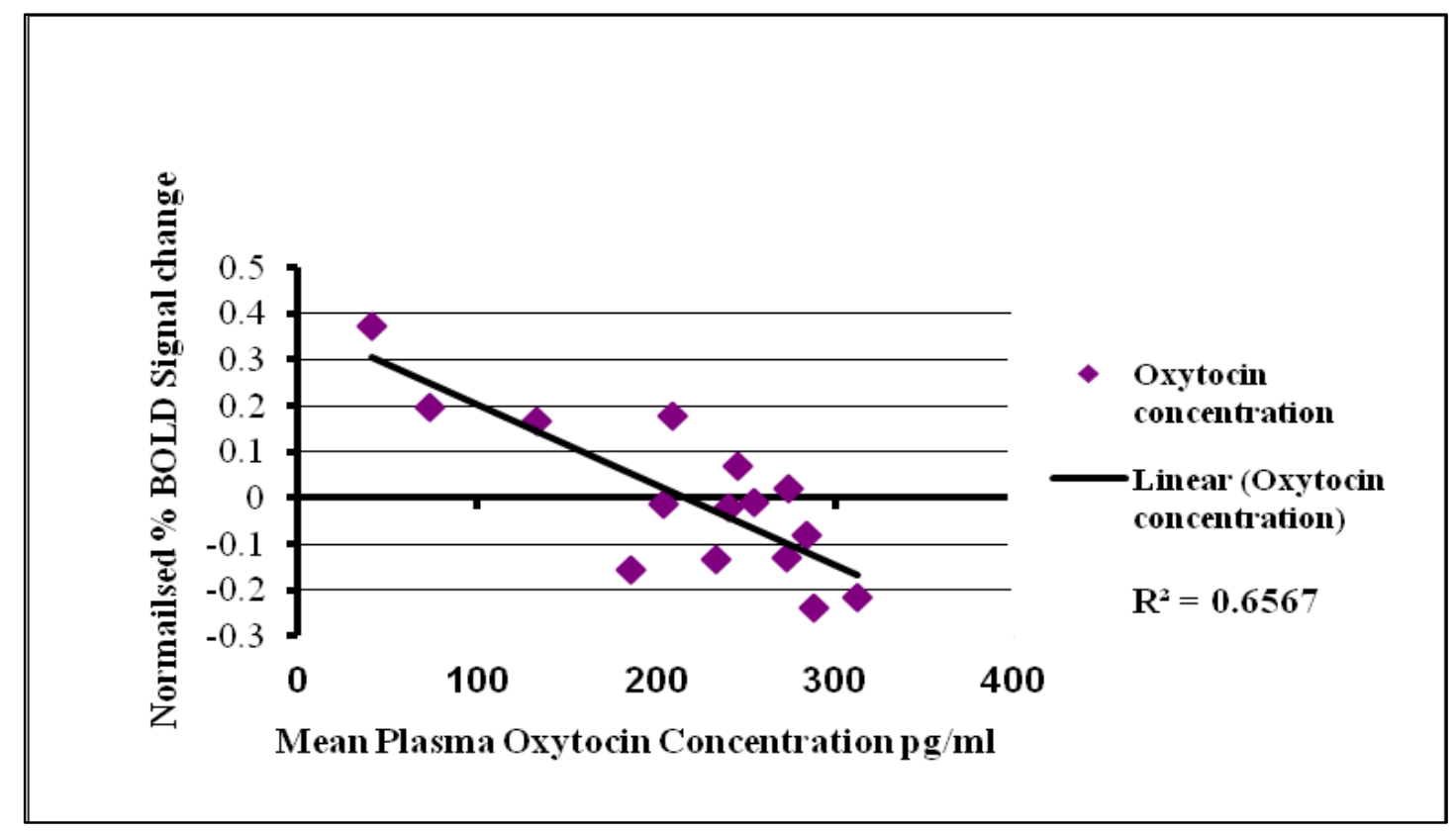

Figure 2. Correlation between BOLD activation in the right superior temporal gyrus (STG) (in response to 'own neutral as compared to unknown neutral infant stimuli') and postinteraction plasma oxytocin among high sensitivity mothers $(\mathrm{N}=15)$. 


\section{APPENDIX}

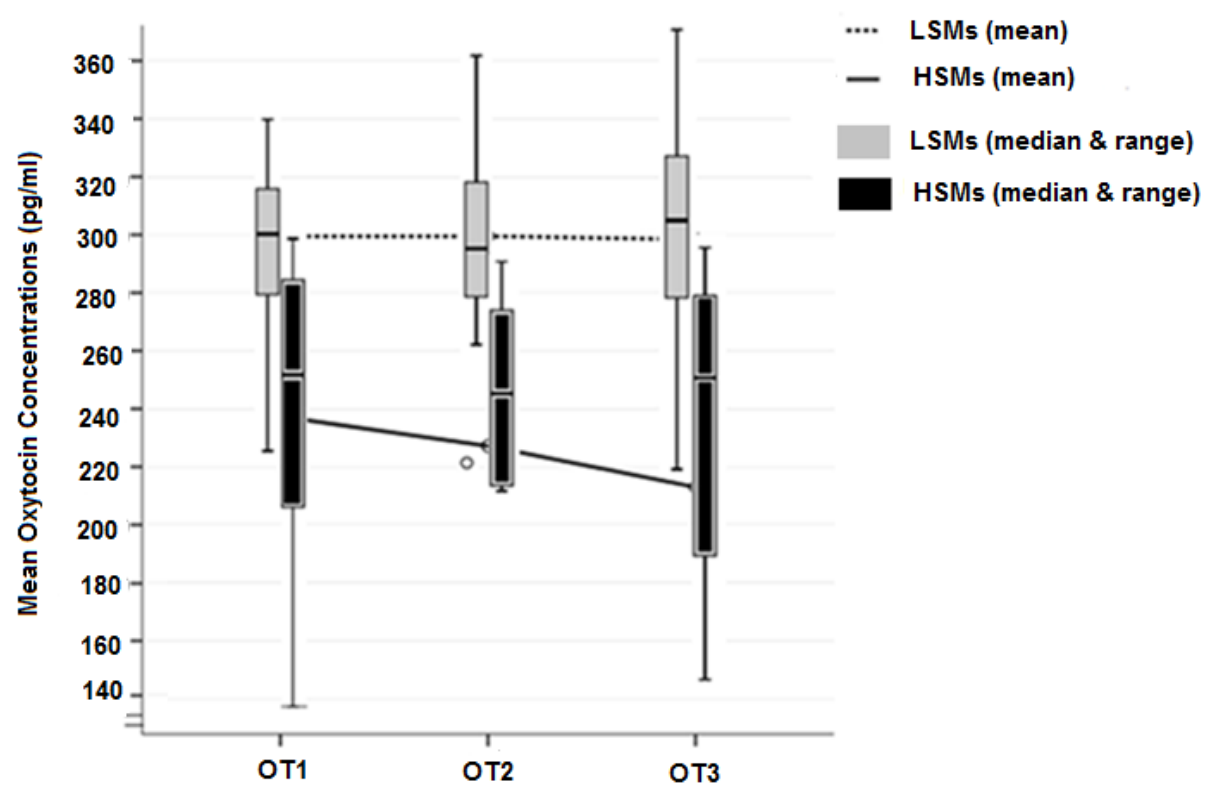

Figure. Means and box plots for plasma oxytocin measured before (OT1) and after motherinfant interaction (OT2 and OT3) among the high sensitivity mothers (HSMs, $\mathrm{N}=15$ ) and low sensitivity mothers (LSMs, $\mathrm{N}=14$ ), controlling for household income. Key: Dashed line represents the means of the three OT assessments among LSMs, and solid line represents means of the three assessments among the HSMs. 
Table. Significant BOLD signals for main effect in response to own versus unknown infant stimuli (combined all affects) among the whole sample $(\mathrm{N}=30)$

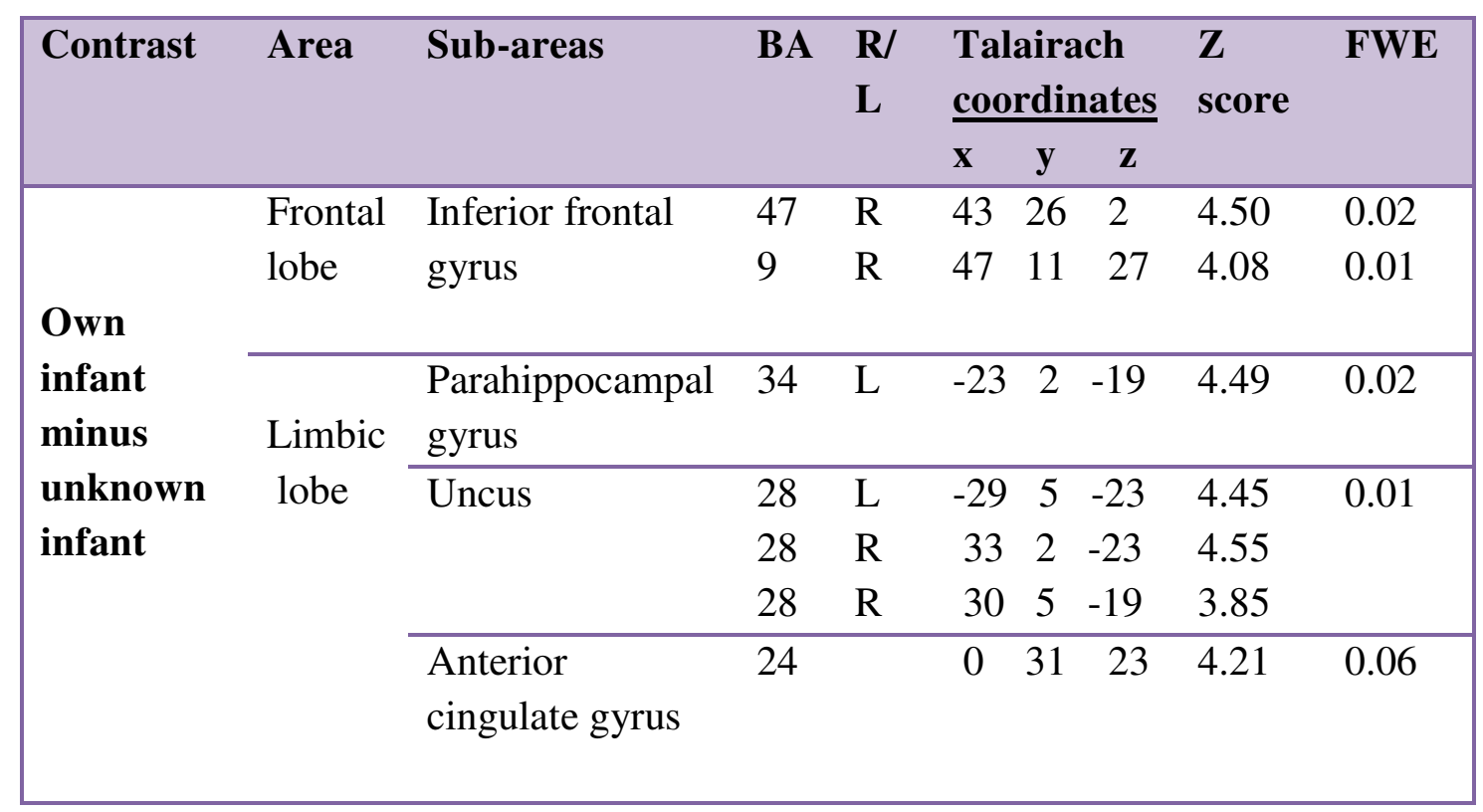

Note. All significant whole brain (Family Wise Error $(\mathrm{FWE}) \leq 0.05)$ corrected for multiple comparison. BA: Brodmann's area; L: Left, R: Right. 
30.6.16 